\title{
ACCELERATION OF PARTICLES IN A CONSTANT MAGNETIC FIELD AND AN ELECTRIC FIELD PERPENDICULAR TO IT, INCREASING WITH TIME
}

\author{
G.N. Kichigin \\ Institute of Solar-Terrestrial Physics SB RAS, \\ Irkutsk, Russia, king@isz.irk.ru
}

\begin{abstract}
The paper addresses the problem of acceleration of particles in a constant, uniform magnetic field of magnitude $B$ and a uniform electric field perpendicular to it, which slowly increases with time. Assuming that the electric field grows linearly up to the maximum value $E_{\mathrm{m}}=B$, approximate analytical relations have been found which determine the particle velocity dependence on the acceleration time. The particles are shown to accelerate for the entire time of the increase in the electric field to a certain final energy, whose value depends on the acceleration rate. It has been established that the lower the acceleration rate, the greater the limiting energy. In the case when the ratio $E_{m} / B<0.9$, using
\end{abstract}

the solution method proposed by Alfvén in the drift approximation, an analytical solution of the relativistic equation of particle motion has been obtained. The results can be used to find the energy of particles in various pulsed processes in space plasma.

Keywords: particle acceleration, crossed electric and magnetic fields, constant magnetic field, timevarying electric field; space plasma.

\section{INTRODUCTION}

In collisionless space plasma, charged particles are mainly accelerated in electromagnetic fields existing in the plasma. One of the important cases is the particle acceleration in stationary magnetic and timevarying electric fields specific to different pulsed processes in the space environment. Such situations occur in flare processes in the solar chromosphere, interplanetary plasma, Earth's magnetosphere and ionosphere. Typically, the time-varying electric field is the so-called polarization field arising from plasma inflow into regions with a curved magnetic field. A well-known example is the electric field of polarization occurring due to separation of oppositely charged particles when plasma stream limited in space moves in the toroidal magnetic field [Morozov, Solov'ev, 1963; Alfvén, Falthammar, 1963; Lindberg, Kristoferson, 1971; Lindberg, 1978; Alfvén, 1981]. Evidence on the occurrence of such fields was obtained in the last century during laboratory experiments, performed by Lindberg [Lindberg, Kristoferson, 1971; Lindberg, 1978], at collisionless plasma ejection into a curved magnetic field.

Time variation of the electric field in magnetized space plasma has a great effect on modulation of cosmic rays (CRs) when they propagate in the heliosphere. The CR modulation effects are described using an approach which assumes that electromagnetic fields in the heliosphere are regular and their structure is adequately described by the Parker model [Parker, 1963]. In this situation, a determining factor in modulation of CRs is a change in their energy when they move in regular electromagnetic fields of the heliosphere. The energy change, in turn, depends on strength and nature of electric fields in the interplanetary medium and on the time of interaction of particles with these fields [Dvornikov,
Sdobnov, 2002]. A comprehensive survey into the CR modulation due to various factors associated with electromagnetic fields of the heliosphere has been carried out in [Dvornikov et al., 2013], where it is shown that under this approach theoretical and experimental CR modulation characteristics are reasonably consistent.

This paper for crossed fields — stationary magnetic and time-varying electric fields - presents solutions of relativistic equations of particle motion, which can be used for finding energy of accelerated particles in different pulsed processes in space plasma and, in particular, for analyzing CR modulation in the heliosphere.

\section{FORMULATION OF THE PROBLEM AND INITIAL EQUATIONS}

Consider motion of a charged particle in a stationary uniform magnetic field $B$ whose vector is directed along the Z-axis, and in a time-varying uniform electric field $E(t)$ directed along the $\mathrm{Y}$-axis. The equations describing the dynamics of particles in such fields in the relativistic case have the form

$$
\frac{d \mathbf{p}}{d t}=q \mathbf{E}+q[\mathbf{v B}] / c, \quad m c^{2} \frac{d \gamma}{d t}=q \mathbf{E v} .
$$

Here, $\mathbf{E}$ and $\mathbf{B}$ are vectors of electric and magnetic fields; $\mathbf{v}, \mathbf{p}$ are vectors of particle velocity and momentum respectively; $q, m$ are particle charge and rest mass; $c$ is the speed of light, $\gamma$ is the dimensionless total particle energy, normalized to the particle energy at rest $m c^{2}$. Hereafter, $[\mathbf{v B}]$ is the vector product. Since the electric and magnetic fields are mutually perpendicular, we have free motion of particles along the magnetic field at a constant velocity and two-dimensional motion in a plane perpendicular to the magnetic field. In this plane, Equations (1) in components take the form 


$$
\begin{aligned}
& \frac{d p_{x}}{d t}=q v_{y} B / c, \frac{d p_{y}}{d t}=q E-q v_{x} B / c, \\
& m c^{2} \frac{d \gamma}{d t}=q E v_{y} .
\end{aligned}
$$

Here, $v_{x}, v_{y}, p_{x}, p_{y}$ are $x$ and $y$ components of particle velocity and momentum respectively. Introduce dimensionless variables: $\tau=\omega_{B} t-$ dimensionless time, where $\omega_{B}=q B /(m c)$ is the nonrelativistic cyclotron frequency; $v, w$ are the $x$ and $y$ velocity components normalized to the speed of light.

Below we solve these equations for a particular case when the particle velocity along the magnetic field is zero, and the electric field increases with time linearly: $E(t)=B t / T$, where $T$ is the time it takes the electric field to increase from zero to $B$. It is assumed that the electric field increases so slowly that for the characteristic time equal to the cyclotron period $T_{\mathrm{c}}=2 \pi / \omega_{B}$ the electric field varies slightly in comparison with $B$. This means that $n$ - the number of cyclotron rotation periods of a particle in the magnetic field $B$ for $T-$ should be large: $n=1 /(2 \pi \varepsilon)>>1$, and hence the parameter $\varepsilon=1 /\left(\omega_{B} T\right)=T_{\mathrm{c}} /(2 \pi T)<<1$ should be small. In real situations, an electric field can increase to a maximum value $E_{\mathrm{m}}<B$. In this case, time of the increase $T_{\mathrm{m}}$ is shorter, whereas $\varepsilon=1 /\left(\omega_{B} T_{\mathrm{m}}\right)$ increases.

In the linearly increasing electric field we assume, the equations for velocity and energy components in dimensionless form are as follows

$$
\frac{d(\gamma v)}{d \tau}=w, \quad \frac{d(\gamma w)}{d \tau}=\varepsilon \tau-v, \quad \frac{d \gamma}{d t}=\varepsilon \tau w .
$$

These equations can be reduced to a system of equations only for velocity components

$$
\frac{d v}{d \tau}=w\left(1-v_{\mathrm{d}} v\right) / \gamma, \quad \frac{d w}{d \tau}=\left[v_{\mathrm{d}}\left(1-w^{2}\right)-v\right] / \gamma,
$$

where $v_{\mathrm{d}}=\varepsilon \tau=E / B$ is the dimensionless drift velocity. In the nonrelativistic case $(\gamma=1)$, system (2) has exact analytical solutions, which at the initial condition $v=w=0$ can be written as:

$$
v=\varepsilon(\tau-\sin \tau), w=\varepsilon(1-\cos \tau)
$$

Equations (2) are also solved analytically if $v_{\mathrm{d}}=1$ [Landau, Livshits, 1967]. In this case, Equations (2) have the form

$$
p_{y}=\gamma w=\gamma \frac{d \gamma}{d \tau}=\gamma \frac{d p_{x}}{d \tau}, \quad p_{x}=\gamma v=\gamma-\gamma \frac{d p_{y}}{d \tau} .
$$

Using these formulas, we get

$$
p_{y}=\gamma \frac{d p_{x}}{d \tau}=\gamma \frac{d \gamma}{d \tau}-\gamma \frac{d}{d \tau}\left(\gamma \frac{d p_{y}}{d \tau}\right) .
$$

For the initial conditions $\gamma=1, p={ }_{x} p_{y}=0$, we have

$$
\gamma \frac{d p_{y}}{d \tau}=\gamma-p_{x}=1
$$

Substituting $\gamma=p_{y} /\left(\frac{d \gamma}{d \tau}\right)$ in (4), we obtain the equality $\quad p_{y} \frac{d p_{y}}{d \tau} / \frac{d \gamma}{d \tau}=1, \quad$ that yields $\gamma=p_{y}^{2} / 2+1$.

Substituting $\gamma$ in (4), we get

$$
\gamma \frac{d p_{y}}{d \tau}=\left(\frac{p_{y}^{2}}{2}+1\right) \frac{d p_{y}}{d \tau}=1,
$$

from which we have [Landau, Livshits, 1967]

$$
p_{y}^{3} / 6+p_{y}=\tau,
$$

which suggests a connection between the momentum $p_{y}$ and the acceleration time. The other variables are given by $p_{y}$ :

$$
\begin{aligned}
& \gamma=p_{y}^{2} / 2+1, \quad p_{x}=p_{y}^{2} / 2, \quad v=p_{y}^{2} /\left(2+p_{y}^{2}\right), \\
& w=2 p_{y} /\left(2+p_{y}^{2}\right) .
\end{aligned}
$$

In general, system of equations (2) can be solved only with numerical methods.

\section{NUMERICAL SOLUTION OF SYSTEM OF EQUATIONS (2)}

In the particular case of the linear dependence of $E(t)$ on time, for the numerical solution of the initial equations we have used the Runge - Kutta method. In the calculations, the parameter $\varepsilon$ varied in the range $10^{-}$ ${ }^{2}>\varepsilon>10^{-7}$, with $\varepsilon$ varying in the range of five orders of magnitude. Results of numerical calculations for $\varepsilon=10^{-2}$ and $\varepsilon=10^{-3}$ are presented in Figure 1, which shows time dependences of velocity components. For clarity, time dependences of $v-v_{\mathrm{d}}, w-\varepsilon \gamma_{\mathrm{d}}^{3}$ are shown, where $v_{\mathrm{d}}, \varepsilon \gamma_{\mathrm{d}}^{3}$ are time averaged oscillating values $v$ and $w$. You can see that at the beginning of acceleration the particle velocity is oscillatory, whereas the oscillation period, as must be the case, coincides with the cyclotron one. Obviously, such velocity behavior of particles is associated with their cyclotron rotation in the magnetic field. The calculations have found that as long as the Larmor rotation of particles during their acceleration exists, the relations are adequately fulfilled

$$
v \approx v \approx v_{\mathrm{d}}=\varepsilon \tau, \quad \gamma \approx \gamma_{\mathrm{d}}=\left(1-v_{\mathrm{d}}^{2}\right)^{-1 / 2}, \quad w=\varepsilon \gamma^{3} .
$$

The Figure shows the main feature of these results is that the particle acceleration process is divided into two stages. At the first stage, solutions are oscillatory, and in the range of the $\varepsilon$ values in use this stage lasts over 90 $\%$ of the acceleration time. It is evident that the duration of the first stage increases with decreasing $\varepsilon$, linked to the rate of increase in the electric field, and in the final phase of the oscillatory stage the period of oscillations of velocity components increases, whereas the amplitude of the oscillations decreases. When the amplitude tends to zero, the character of solutions changes abruptly, 


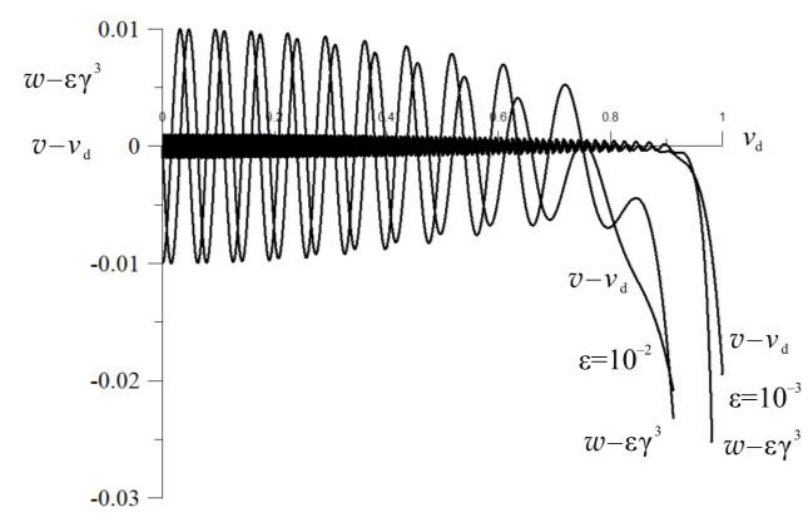

Figure 1. Time dependence (in units of $v_{\mathrm{d}}$ ) of particle velocity components for $\varepsilon=0.01$ and $\varepsilon=0.001$. Differences are given between time oscillating velocity components $v$ and $w$ and their time averaged values $v_{\mathrm{d}}$ and $\varepsilon \gamma^{3}$ respectively

and the second stage - the aperiodic stage of velocity variation with time, at which the time dependence of the components takes the aperiodic monotonous form begins. As revealed in the analysis of the numerical calculation results, both at the first and at the second stage, for the velocity component $w$ the relation $w=\varepsilon \gamma^{3}$, which was taken as a basis, proved to be quite acceptable in accuracy. It is easy to show by the first formula of system (2) that the relation $w=\varepsilon \gamma^{3}$ can be obtained if we set $v v_{\mathrm{d}}=v^{2}, v \approx v_{\mathrm{d}}=\varepsilon \tau$, and that has been done. Further, from $v^{2}=v v_{\mathrm{d}}=v^{2}+w^{2}$, given that in calculations $w / v_{\mathrm{d}}<1$, we have found a more accurate expression for the $x$ velocity component $v=v_{\mathrm{d}}\left(1-w^{2} / v_{\mathrm{d}}^{2}\right)$. Thus, taking into account relation (7) at the first stage and using the method of fitting suitable relations, which can adequately describe the temporal behavior of the velocity components in the numerical calculations at the second stage, we have arrived at the following important conclusion: throughout the time interval of acceleration, where $w / v_{\mathrm{d}}<1$, the following approximate time dependences of particle velocity are considered reasonably adequate in accuracy:

$$
v=\left(v v_{\mathrm{d}}\right)^{1 / 2}, w=\varepsilon \gamma^{3}, v=v_{\mathrm{d}}\left(1-w^{2} / v_{\mathrm{d}}^{2}\right) .
$$

Note that at the oscillatory stage of acceleration all terms in Formula (8) are time average. The values determined from Formula (8) and obtained by numerical calculations roughly coincide.

For example, their maximum difference, observed in the calculations at a time point at which $v_{\mathrm{d}}=0.999999$ (a value close to the finite $\left.v_{\mathrm{d}}=1\right)$, is as follows: 1) for $\varepsilon=10^{-2}\left(v-\left(v v_{\mathrm{d}}\right)^{1 / 2}\right)=-0.016, \quad w-\varepsilon \gamma^{3}=0.19, \quad v-\left(v_{\mathrm{d}}-\right.$ $\left.\left.w^{2} / v_{\mathrm{d}}\right)=0.065 ; 2\right)$ for $\varepsilon=10^{-4}\left(v-\left(v v_{\mathrm{d}}\right)^{1 / 2}\right)=-0.0018$, w$\varepsilon \gamma^{3}=0.054, v-\left(v_{\mathrm{d}}-w^{2} / v_{\mathrm{d}}\right)=0.0048$. It can be seen that as $\varepsilon$ decreases this difference becomes progressively smaller, and the largest at the maximum value of $\varepsilon=10^{-2}$ does not exceed $20 \%$.

Using (8), for the particle energy at the second stage we can get

$$
1 / \gamma_{d}^{2}=1 / \gamma^{2}-\varepsilon^{2} \gamma^{6}
$$

from which it follows that upon completion of the acceleration, i.e. at $v_{\mathrm{d}}=1$, energy has a limiting value $\gamma_{\mathrm{m}}=\varepsilon^{-1 / 4}$. This means that at finite values of $\varepsilon$ the maximum particle energy obtained over the entire acceleration period is limited, and its limiting value is controlled by $\varepsilon$. Dependence of the limiting energy $\gamma_{\mathrm{m}}$ on $\varepsilon$ is plotted in Figure 2. There are two curves, one of which is obtained from numerical calculations, the other is the dependence represented by the formula $\gamma_{\mathrm{m}}=\varepsilon^{-1 / 4}$. Obviously, they differ little, indicating that the approximate solutions found for (8) are absolutely reliable.

Estimate the time interval in which we can use Formula (8). Duration of this interval can be determined from the inequality $w / v_{\mathrm{d}}<1$, which can be represented as $\tau>\gamma^{3}$. In the initial time interval of the acceleration, where $v_{\mathrm{d}} \approx 0, \gamma \approx 1$, this inequality is satisfied for $\tau>1$; and at the end of acceleration, where $\gamma_{\mathrm{m}}=\varepsilon^{-1 / 4}, \tau_{\mathrm{m}}=1 / \varepsilon$, the inequality also holds as $\tau=\tau_{\mathrm{m}}=1 / \varepsilon>\gamma_{\mathrm{m}}^{3}=1 / \varepsilon^{3 / 4}$. Thus, we can assume that the initial time interval $\tau_{\mathrm{n}}$ in which Formulas (8) cannot be used is negligibly small compared to the total time of acceleration $\tau_{\mathrm{m}}\left(\tau_{\mathrm{n}} \sim 1<<\tau_{\mathrm{m}}=1 / \varepsilon\right)$, and in fact Formulas (8) can be applied to the entire time interval of particle acceleration.

In concluding this section, note that at $v_{\mathrm{d}} \rightarrow 1$ it seems possible to make use of solutions (5), (6), and the closer $v_{\mathrm{d}}$ to unity, the greater the confidence in the validity of these formulas. It appears, however, that if $1-v_{\mathrm{d}}<\varepsilon$, the residual time $\tau_{\text {res }}$ during which a particle can still be accelerated is too short: $\tau_{\text {res }}<1$. This means that for the time interval, as derived from (6), there is no appreciable increase in energy $\Delta \gamma$ as $\Delta \gamma<1$, and applying Formulas (5), (6) under favorable conditions of their use $\left(1-v_{\mathrm{d}}<\varepsilon\right)$ unfortunately proves to be inefficient.

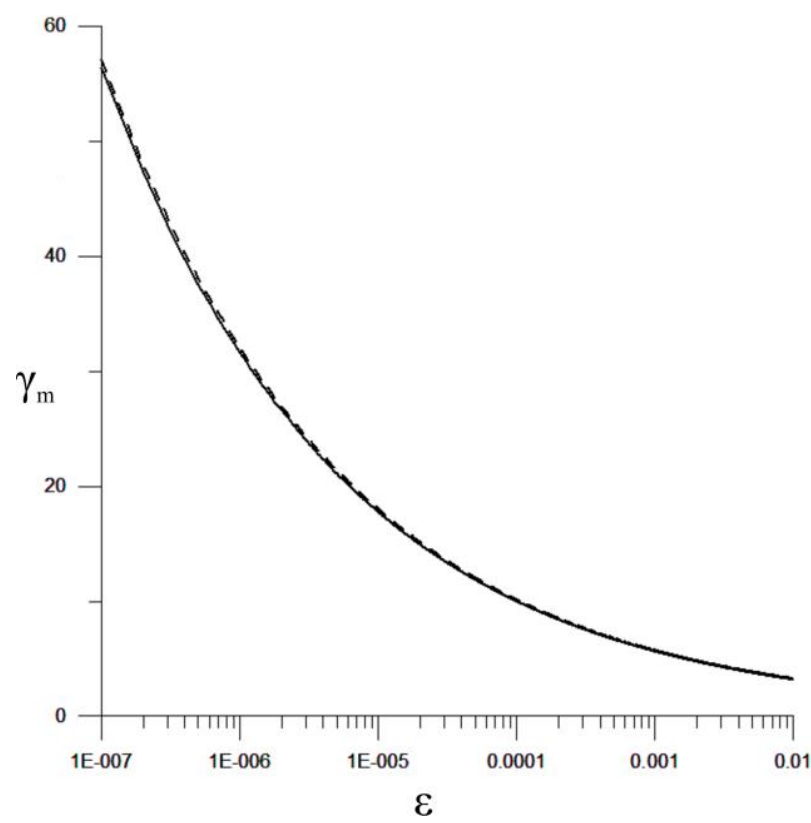

Figure 2. Parameter $\varepsilon$ as a function of limiting energy $\gamma_{\mathrm{m}}$, obtained from numerical calculations (solid curve) and from $\gamma_{\mathrm{m}}=\varepsilon^{-1 / 4}$ (dashed curve) 


\section{SOLVING EQUATION OF PARTICLE MOTION USING ALFVÉN'S METHOD AT THE OSCILLATORY STAGE}

At the oscillatory stage, as it follows from numerical calculations, the particle dynamics is determined by their drift motion in crossed electric and magnetic fields. To solve problems with such behavior of charged particles, Alfvén once proposed a method that may be considered usable in this case. This method appears to be very helpful for the problem to solve.

Alfvén's method is described in detail in [Dorman et al., 1971]. To apply it confidently, we had to find the $\varepsilon$ value such that the drift approximation, whose fulfillment is necessary for Alfvén's method, would be still valid. As a limiting value of $\varepsilon$, for which, let us agree, Alfvén's method is still applicable, we take $\varepsilon=0.01$ (with $n \approx 16$, i.e. the field $E$ reaches a value $B$ for a time span equal to 16 cyclotron periods). In our opinion, if $E$ increases more rapidly, the use of Alfvén's method for solving this problem is already in doubt.

Following Alfvén's method, to solve the equation of particle motion as the first step represent the particle velocity as a sum of two velocities - the drift velocity $\mathbf{v}_{\mathrm{d}}=c[\mathbf{E B}] / B^{2}$ and the velocity $\mathbf{v}_{1}: \mathbf{v}=\mathbf{v}_{\mathrm{d}}+\mathbf{v}_{1}$. Then the motion equation takes the form

$$
\frac{d\left(m \gamma \mathbf{v}_{\mathbf{1}}\right)}{d t}=q\left[\mathbf{v}_{1} \mathbf{B}\right] / c-\frac{d\left(m \gamma \mathbf{v}_{\mathrm{d}}\right)}{d t} .
$$

In the next approximation, assuming in the second step that the electric field slowly increases with time, take into account the effect of the weak force $\mathbf{f}=-\frac{d\left(m \gamma \mathbf{v}_{\mathrm{d}}\right)}{d t}$, acting on the particle. Let $\mathbf{v}_{1}=\mathbf{v}_{2}+\mathbf{v}_{\mathrm{P}}$, where $\mathbf{v}_{\mathrm{P}}=c[\mathbf{f B}] /\left(q B^{2}\right)$ is the particle drift velocity under the action of the magnetic field $\mathbf{B}$ and the force $\mathbf{f}$. Given that the vectors $\mathbf{f}$ and $\mathbf{B}$ are mutually perpendicular, this yields

$$
\frac{d\left(m \gamma \mathbf{v}_{2}\right)}{d t}=q\left[\mathbf{v}_{2} \mathbf{B}\right] / c-\frac{d\left(m \gamma \mathbf{v}_{\mathrm{P}}\right)}{d t}
$$

In the third step, putting $\frac{d\left(m \gamma \mathbf{v}_{\mathrm{P}}\right)}{d t} \approx 0$, we find the solution of the motion equations as $\mathbf{v}=\mathbf{v}_{2}+\mathbf{v}_{\mathrm{d}}+\mathbf{v}_{\mathrm{P}}$. If we confine ourselves to this approximation, the solution obtained will characterize the particle motion round the Larmor circle at a velocity of $\mathbf{v}_{2}$ and its drift in two directions: the electric drift at a velocity $\mathbf{v}_{\mathrm{d}}$ in a direction perpendicular to the electric and magnetic fields and the drift at a velocity $\mathbf{v}_{\mathrm{P}}$ in the direction of the electric field vector $\mathbf{E}$. Note that in a linearly increasing electric field in this step the solution $\mathbf{v}=\mathbf{v}_{2}+\mathbf{v}_{\mathrm{d}}+\mathbf{v}_{\mathrm{P}}$, we have obtained using Alfvén's method, for nonrelativistic particles satisfies the equation of their motion (about the existence of such a solution in the nonrelativistic case see [Morozov, Solov'ev, 1963]). As you can see, the solution for the velocity components $v=\varepsilon \tau, w=\varepsilon$ (at $\mathbf{v}_{2}=0$ ) found by Alfvén's method and satisfying the equation of particle motion, is a time averaged exact solution of (3).
The approximate solution we have derived suggests that due to the particle drift at a velocity $\mathbf{v}_{\mathrm{P}}$ the total particle energy $\gamma m c^{2}$ will change; it can be found from the relation

$$
\begin{aligned}
& m c^{2} \frac{d \gamma}{d t}=q \mathbf{E} \mathbf{v}_{\mathrm{P}}=q \mathbf{E} c[\mathbf{f B}] /\left(q B^{2}\right)= \\
& =-m q c \mathbf{E}\left[\frac{d\left(\gamma \mathbf{v}_{\mathrm{d}}\right)}{d t} \mathbf{B}\right] / B^{2} .
\end{aligned}
$$

Substituting $\mathbf{v}_{\mathrm{d}}=c[\mathbf{E B}] / B^{2}$ in it, for the vector product $\left[\frac{d\left(\gamma \mathbf{v}_{\mathrm{d}}\right)}{d t} \mathbf{B}\right]$ we get

$$
\left[\frac{d\left(\gamma \mathbf{v}_{\mathrm{d}}\right)}{d t} \mathbf{B}\right]=-c\left(\gamma \frac{d \mathbf{E}}{d t}+\mathbf{E} \frac{d \gamma}{d t}\right) / B^{2} .
$$

Hence, in the increasing electric field the energy increment can be determined from the equation

$$
2\left(1-v_{\mathrm{d}}^{2}\right) \frac{d \gamma}{d t}=\gamma \frac{d v_{\mathrm{d}}^{2}}{d t} .
$$

In the general case, for the approximation of interest $v_{\mathrm{d}}<1$ we solve Equation (9) as follows

$$
\gamma=\left(1-v_{\mathrm{d}}^{2}\right)^{-1 / 2}
$$

In the particular case, for $v_{\mathrm{d}}<<1$ Equation (9) is solved as follows

$$
\gamma=\exp \left(v_{\mathrm{d}}^{2} / 2\right)
$$

Thus, for small $v_{\mathrm{d}}<<1$, according to (11), the total energy increases exponentially as a function of $v_{\mathrm{d}}$. With moderate $v_{\mathrm{d}}$, but when $v_{\mathrm{d}}<1$, the energy increase is determined by Formula (10), said formally, as follows from (10), for $v_{\mathrm{d}} \rightarrow 1$ the energy $\gamma$ increases indefinitely. When $v_{\mathrm{d}} \rightarrow 0$, (10) and (11) give the same result

$$
\gamma \approx 1+v_{\mathrm{d}}^{2} / 2 \text {. }
$$

From (10) it follows that the solution of relativistic equation of motion (1) is the magnitude of the drift velocity: $\mathbf{v}=\mathbf{v}_{\mathrm{d}}$. If we substitute the formally found solution $\mathbf{v}=\mathbf{v}_{\mathrm{d}}$ into motion equation (1), we obtain that it is satisfied if the condition $\frac{d\left(m \gamma \mathbf{v}_{\mathrm{d}}\right)}{d t}=0$. holds. The fulfillment of the condition means that in this case $\nu=v_{\mathrm{d}}, w=\varepsilon \gamma^{3}=\frac{d\left(m \gamma v_{\mathrm{d}}\right)}{d t}=0$, and $\varepsilon=0$. We can conclude that with $\varepsilon \rightarrow 0$ the complete particle acceleration cycle is accomplished at the oscillatory stage, i.e. during drift motion of particles in which the time dependence of particle energy is determined by Formula (10).

\section{APPLICABILITY OF THE RESULTS FOR PULSED PROCESSES IN THE HELIOSPHERE}

In this section, we give some ideas about how the results can be used. Let us address the problem of CR modulation in the heliosphere, mentioned in Introduc- 
tion. Two problems should be considered here: 1) the change in CR energy when passing a heliospheric region whose electric field varies with time; 2) to determine the mechanism behind the time-varying electric field in the heliosphere. For problem 1 we assume that the density of CR particles (mostly protons) is negligible with respect to the particle density in heliospheric plasma. This condition allows us to analyze the dynamics of charged particles in electromagnetic fields in the one-particle approximation, presented in this paper. Thus, the results obtained in this work can be confidently used to solve the problems at hand. For problem 2 we suppose that the increasing electric field is induced during motion of spatially bounded plasma streams ejected from the Sun and moving in the curved magnetic field of the heliosphere (Parker field).

Consider such a scenario. Let there be a plasma region in which on the background of a mean magnetic field $B$ an increasing electric field perpendicular to the magnetic field appears. We are interested in the energy obtained by charged particles present in this region. In such a situation, to use the results, obtained in this work, for estimating the energy, it is necessary to know first the time $t_{\mathrm{m}}$ it takes the electric field to increase from zero to a maximum value $E_{\mathrm{m}}$. Then the parameter $\varepsilon$, associated with the rate of the field increase, has the form $\varepsilon=E_{\mathrm{m}} /\left(B \omega_{B} t_{\mathrm{m}}\right)$. Assume further that in the direction of the particle's electric drift the size of the region we identified should be at least $c t_{\mathrm{m}}$, and moreover the conditions $\omega_{B} t_{\mathrm{m}}<<1, E_{\mathrm{m}} / B \leq 1$ should also be met. Then, knowing $\varepsilon$, for $E_{\mathrm{m}} / B \approx 1$ from $\gamma_{\mathrm{m}}=\varepsilon^{-1 / 4}$ we can estimate the limiting energy transferred to a particle throughout the acceleration period. If $E_{\mathrm{m}} / B<0.9$, to assess the limiting energy we can use the formula $\gamma=\left(1-v_{\mathrm{d}}^{2}\right)^{-1 / 2}$, where $v_{\mathrm{d}}=E_{\mathrm{m}} / B$.

Let us discuss the basic processes in the heliosphere, which lead to $\mathrm{CR}$ acceleration. According to [Dvornikov et al., 2013], in regular electromagnetic fields of the heliosphere the acceleration of $\mathrm{CR}$ energetic particles is generally due to three reasons: 1) particle drift toward the heliospheric electric field; 2) time variation of the magnetic field (betatron acceleration); 3) time variation of the electric field. In the first case, a charged particle can gain energy up to $200 \mathrm{MeV}$ [Dvornikov et al., 2013]. In the other two cases, to assess the particle energy resulting from acceleration, we turn to specific events occurring in the heliosphere in OctoberNovember 2003. These events have been analyzed in [Dvornikov et al., 2013] (see Figure 3 therein). At betatron acceleration, the CR energy change in these events is maximum when $\beta=2$. For CRs entering the alternating magnetic field of the heliosphere with an energy 100 $\mathrm{MeV}-1 \mathrm{GeV}$, the maximum particle energy due to the betatron acceleration varies from 40 to $400 \mathrm{MeV}$. To estimate the energy change in the case of increasing electric field, as follows from aforesaid Figure 3, the value $\alpha=E^{2} / B^{2}$ varied from 0.2 to 0.24 . Hence, the kinetic energy of particles $K=m c^{2}\left[\left(1-E^{2} / B^{2}\right)^{-1 / 2}-1\right]$ varied from 110 to $150 \mathrm{MeV}$. You can see that all the three acceleration mechanisms make a comparable and quite significant contribution to the CR energy change.

\section{MAIN RESULTS AND CONCLUSIONS}

An approximate analytical solution has been found for the relativistic equation of particle motion in the spatially uniform electromagnetic field, whose structure is defined by the stationary magnetic field $B$ and by the electric field strictly perpendicular to it, which linearly increases with time from zero to a maximum value $E_{\mathrm{m}}=B$. The solution is represented by fairly simple formulas (8), which define the dependence of particle velocity on acceleration time. For the entire period of electric field increase to $B$, particles are shown to accelerate to a finite energy that is determined by $\varepsilon$, associated with the rate of electric field increase, and the smaller the rate, the higher the limiting energy.

For the range of $\varepsilon$ change $\left(10^{-2}>\varepsilon>10^{-7}\right)$ we take here, it has been found that if $E_{\mathrm{m}} / B<0.9$, the dynamics of relativistic particles is determined by the relations obtained from solutions of the motion equation by the method proposed by Alfvén. In this case, the maximum kinetic energy

$$
K_{\mathrm{m}}=m c^{2}\left[\left(1-E_{\mathrm{m}}^{2} / B^{2}\right)^{-1 / 2}-1\right] .
$$

We believe that these results are important for understanding the dynamics of charged particles when moving in electromagnetic fields and are of general physical interest. Formulas (8) and (10) can be used to find particle energy in various pulsed processes in space plasma, which occur in an electromagnetic field similar to that considered in this work.

I am very grateful to reviewers for their helpful comments.

The work was performed under Project II.16.1.3 of SB RAS Basic Research Program II.16.1 (research priority II.16).

\section{REFERENCES}

Alfvén H. Kosmicheskaya plazma. Moscow, Mir Publ., 1983, 216 p. (In Russian). English Edition: Alfvén H. Cosmic Plasma. Dordrecht, Reidel Publishing Company, 1981, 213 p.

Alfvén H., Falthammar K.G. Kosmicheskaya elektrodinamika. Moscow, Mir Publ., 1967. 206 p. English Edition: Alfvén H., Falthammar K.G. Cosmical Electrodynamics. Intern. Ser. of Monographs on Physics. Oxford, Clarendon Press, 1963, $260 \mathrm{p}$.

Dorman L.I., Smirnov V.S., Tyasto M.I. Kosmicheskie luchi $v$ magnitnom pole Zemli [Cosmic Rays in the Earth's Magnetic Field]. Moscow, Nauka Publ., 1971, 399 p. (In Russian).

Dvornikov V.M., Sdobnov V.E. Variations in the rigidity spectrum and anisotropy of cosmic rays at the period of Forbush effect on 12-15 July 1982. International $J$. Geomagnetism and Aeronomy. 2002, vol. 3, no. 3, pp. 217-228.

Dvornikov V.M., Kravtsova M.V., Sdobnov V.E. Diagnostics of the electromagnetic characteristics of the interplanetary medium based on cosmic ray effects. Geomagnetism and Aeronomy. 2013, vol. 53, iss. 4, pp. 430-440. DOI: $10.1134 /$ S0016793213040075. 
Landau L.D., Livshits E.M. Teoriya polya [Field Theory]. Moscow, Nauka Publ., 1967, 460 p. (In Russian).

Lindberg L. Plasma flow in a curved magnetic field. Astrophys. Space Sci. 1978, vol. 55, p. 203-225. DOI: 10.1007/ BF00642589.

Lindberg L., Kristoferson L. Reverse deflection and contraction of a plasma beam entering a curved magnetic field. Cosmic Electrodynamics. 1971, vol. 2, pp. 305-380.

Morozov A.I., Solov'ev. L.S. Motion of charged particles in electromagnetic fields. Voprosy teorii plazmy [Reviews of Plasma Physics]. Moscow, Gosatomizdat Publ., 1963. Iss. 2. P. 177-255. (In Russian). English Edition: Morozov A.I.
Solov'ev L.S. Reviews of Plasma Physics, Vol. 2. Authorized translation from the Russian by Herbert Lashinsky, University of Maryland, USA. Published by Consultants Bureau, New York, 1966, $201 \mathrm{p}$.

Parker E.N. Interplanetary dynamical processes. New York, London, Interscience Publ., 1963, 284 p.How to cite this article

Kichigin G.N. Acceleration of particles in a constant magnetic field and an electric field perpendicular to it, increasing with time. Solar-Terrestrial Physics. 2021. Vol. 7. Iss. 2. P. 22-27. DOI: $10.12737 /$ stp-72202103 